\title{
A closed-tube methylation-sensitive high resolution melting assay (MS-HRMA) for the semi-quantitative determination of CST6 promoter methylation in clinical samples
}

\author{
Lampros Dimitrakopoulos ${ }^{1}$, Panagiotis A Vorkas ${ }^{1,3}$, Vasilis Georgoulias ${ }^{2}$ and Evi S Lianidou ${ }^{1 *}$
}

\begin{abstract}
Background: CST6 promoter is highly methylated in cancer, and its detection can provide important prognostic information in breast cancer patients. The aim of our study was to develop a Methylation-Sensitive High Resolution Melting Analysis (MS-HRMA) assay for the investigation of CST6 promoter methylation.

Methods: We designed primers that amplify both methylated and unmethylated CST6 sequences after sodium bisulfate (SB) treatment and used spiked control samples of fully methylated to unmethylated SB converted genomic DNA to optimize the assay. We first evaluated the assay by analyzing 36 samples (pilot training group) and further analyzed 80 FFPES from operable breast cancer patients (independent group). MS-HRMA assay results for all 116 samples were compared with Methylation-Specific PCR (MSP) and the results were comparable.

Results: The developed assay is highly specific and sensitive since it can detect the presence of $1 \%$ methylated CST6 sequence and provides additionally a semi-quantitative estimation of CST6 promoter methylation. CST6 promoter was methylated in 39/80 (48.75\%) of FFPEs with methylation levels being very different among samples. MS-HRMA and MSP gave comparable results when all samples were analyzed by both assays.

Conclusions: The developed MS-HRMA assay for CST6 promoter methylation is closed tube, highly sensitive, cost-effective, rapid and easy-to-perform. It gives comparable results to MSP in less time, while it offers the advantage of additionally providing an estimation of the level of methylation.
\end{abstract}

Keywords: Methylation-sensitive high-resolution melting analysis, Cystatin M, CST6, DNA methylation, Breast cancer, Methylation specific PCR

\section{Background}

DNA methylation is one of the most frequent epigenetic events in the mammalian genome that usually occurs in regions rich in CG dinucleotides. Alterations in DNA methylation are very common in cancer cells; many tumor suppressor genes which are normally unmethylated, when they undergo aberrant DNA methylation are silenced and as a consequence they are not expressed [1]. In particular, hypermethylation has been reported as an early event in breast cancer [2], frequently leading to gene silencing

\footnotetext{
*Correspondence: lianidou@chem.uoa.gr

'Laboratory of Analytical Chemistry, Department of Chemistry, University of Athens, Athens 15771, Greece

Full list of author information is available at the end of the article
}

through methylation of CpG-rich regions near the transcriptional start sites of genes that regulate important cell functions [3]. DNA methylation is believed to be an early event in the process of cancer development and progression since tumor suppressor genes are frequently inactivated at very early stages in human cancer. Thus, DNA methylation is considered as a promising biomarker for early detection and prognosis estimation in cancer patients $[4,5]$.

Sodium bisulfite (SB) modification of DNA is necessary for DNA methylation assays that are based on PCR amplification, since DNA polymerase does not recognize methylated nucleotides, and as a result methylation information is lost during amplification. Through bisulfite treatment

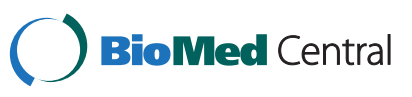


this information is maintained, since unmethylated cytosines are transformed into uracils, while 5-methylcytosines remain unaffected. There are two different approaches, which allow DNA methylation analysis through PCR amplification of SB modified DNA. The first approach is based on design of primers that specifically amplify methylated or unmethylated templates, and is adopted by methylation specific PCR (MSP) and quantitative MSP. The second approach is based on primers that amplify a region of the desired template including $\mathrm{CpG}$ islands, no matter what its methylation status is. In this case, Methylation Independent PCR (MIP) is firstly performed and information on the methylation status of that region is obtained through postPCR analyses techniques like bisulfite sequencing, restriction digestion, single-strand conformation analysis, and high-resolution melting [6].

High-Resolution Melting Analysis (HRMA) firstly introduced in 2003 [7] has several advantages for clinical analysis, since it is a closed-tube, probe-free technique, rapid, simple, cost-effective and non-destructive. Initially developed for mutation scanning and genotyping studies [8-12], high-resolution melting technology can be useful for the detection of methylation as well. Recently, the development of a new generation of melting instrumentation and the introduction of highly sensitive fluorescent dye chemistries, allowed the development of MethylationSensitive High-Resolution Melting Analysis (MS-HRMA). MS-HRMA is based on the different melting profiles of unmethylated and methylated PCR products, due to their different sequence composition (CG content) [6]. MS-HRMA is characterized by high sensitivity, reproducibility and accuracy, while it is a closed tube method less prone to contamination problems [13].

Cystatin M or E/M (encoded by the CST6 gene) is an endogenous inhibitor of lysosomal cysteine proteases that functions to protect cells against uncontrolled proteolysis [14]. Cystatin M was first identified and cloned by Sotiropoulou et al. by differential RNA display as a transcript that was significantly down-regulated in metastatic breast cancer cells when compared to primary breast cancer cells [15]. Later, the same protein was identified and cloned independently from embryonic lung fibroblasts and was named Cystatin E [16]. Cystatin E/M is a low molecular mass protein sharing 27-32\% homology with other cystatins. Cystatin $M$ has been assigned to chromosome region 11q13 [17], which is the site of loss of heterozygosity (LOH) in several cancer types and believed to harbor tumor suppressor genes. Cystatin $M$ was shown to directly inhibit the activity of cathepsins $\mathrm{B}, \mathrm{V}$, and $\mathrm{L}$ $[18,19]$. In addition, cystatin $M$ controls the activity of legumain, which is a known oncogene and an indicator of poor prognosis in colorectal and breast cancer but was also found overexpressed in the majority of human solid tumors $[20,21]$. Thus, imbalance between proteases and their inhibitors cystatins can lead to tumor development, invasion and metastasis [22]. Analysis of the CST6 gene shows a single $\mathrm{CpG}$ island with many potential methylation sites in the promoter and the exon 1 of the gene ( $\sim 64$ CpGs in a 507 bp segment) [23] and it was recently shown that this region is a target for DNA methylation, which results in loss of cystatin $M$ expression in breast cancer lines and breast carcinomas [23-25].

We have previously demonstrated that CST6 is hypermethylated in breast cancer tissues and that CST6 promoter methylation provides important prognostic information in patients with operable breast cancer [26]. Moreover we have recently shown that CST6 is epigenetically silenced in Circulating Tumor Cells (CTC) isolated from peripheral blood of operable and metastatic breast cancer patients [27]. Herein, we report a novel closedtube MS-HRMA assay for the semi-quantitative determination of CST6 promoter methylation in clinical samples. Moreover, performance of the developed CST6 MS-HRMA assay is compared to the performance of our previously described methylation specific PCR for CST6.

\section{Methods}

\section{Patients and samples}

Our study material consisted of a total of 116 clinical samples: a) one pilot testing group, consisting of 36 samples: 10 paired breast cancer and 10 adjacent histologically normal non-cancerous tissues, 7 histologically cancer-free specimens obtained from healthy women during reduction mammoplasty, and 9 breast fibroadenomas (included as a separate benign tumor group) and b) one independent cohort consisting of 80 formalin fixed paraffinembedded (FFPE) breast carcinomas, obtained from patients with operable breast cancer from the Department of Medical Oncology, University Hospital of Heraklion Crete. All samples were collected at diagnosis and all patients gave their informed consent to participate in the study which has been approved by the Ethical and Scientific Committees of our Institution. Tissue sections of $10 \mu \mathrm{m}$ containing $>80 \%$ of tumor cells were used for DNA extraction and for MS-HRM analysis. Genomic DNA (gDNA) from paraffin tissues was isolated with the High Pure PCR Template Preparation kit (Roche, Germany). DNA concentration was determined in the Nanodrop ND-1000 spectrophotometer (Nanodrop Technologies, USA). Before proceeding to the sodium bisulfite conversion and MSP reaction steps, the genomic DNA integrity of all our clinical samples was assessed by amplifying BRCA1 exon 20 for mutation analysis by using the same primers as previously described [28].

\section{Sodium bisulfite conversion}

$1 \mu \mathrm{g}$ of extracted DNA was modified with sodium bisulfite (SB), in order to convert all unmethylated, but not 
methylated-cytosines to uracil. Bisulfite conversion was carried out using the EZ DNA Methylation Gold Kit (ZYMO Research Co., Orange, CA), according to the manufacturer's instructions. The converted DNA was stored at $-70^{\circ} \mathrm{C}$ until used. In each sodium bisulfite conversion reaction, $\mathrm{dH}_{2} \mathrm{O}$ and breast cancer cell line MCF-7 were included as a negative and positive control, respectively.

\section{Controls}

Human placental genomic DNA (gDNA; Sigma-Aldrich) and Universal Methylated Human DNA Standard (ZYMO Research Co., Orange, CA), were used as fully unmethylated and fully methylated controls respectively. Both controls underwent sodium bisulfite conversion, and a series of synthetic controls containing $0 \%, 1 \%$, $10 \%, 50 \%$ and $100 \%$ methylated DNA were prepared by spiking the fully methylated DNA control into the unmethylated. These synthetic methylated DNA controls were used for the evaluation of the sensitivity of the assay and the semi-quantitative estimation of CST6 methylation in our clinical samples.

\section{Methylation sensitive high resolution melting (MS-HRM) In silico primer design}

The primer set was designed in silico, using the PrimerPremier 5 software (Premier Biosoft International, USA), and synthesized by FORTH (Heraklion, Greece). During PCR the methylated and unmethylated templates have to be amplified equally so as the percentage of the methylated products reflects the percentage in the original sample. In low annealing temperatures bias favor the unmethylated template [29]. Therefore, the annealing temperature is critical. In order to reverse those PCR bias, improve the sensitivity of the assay and ensure that only SB converted DNA is amplified the primer set was designed according to the guidelines of Wojdacz et al. $[6,30-33]$. The sequence for the forward primer is 5'-GGTTTAGCGTTAGCGGGAGGTT-3' and for the reverse primer is $5^{\prime}$-AACTCGACACTCACGACTCTAAA AACTC-3'. The PCR amplicon consists of $79 \mathrm{bp},(+9$ up to +87 ; +1 being the transcriptional start site of CST6 gene) [34]. The reverse primers are within the same region that was used for the nested MSP in the same samples as previously described [26]. The exact position of CGs in the CST6 gene and the MS-HRMA and MSP primers used in this study are shown in Figure 1.

\section{PCR}

Real-time PCR was performed in the LightCycler ${ }^{\circledR} 480$ instrument (Roche Applied Science, Germany) using 96well plates (Roche Applied Science, Germany). Extensive optimization experiments were performed in order to maximize PCR amplification efficiency, including PCR program parameters, $\mathrm{Mg}^{2+}$, primer and template concentrations. In addition optimization for the annealing temperature in order to reverse PCR bias as described above was carried out. $1 \mu \mathrm{L}(\sim 100 \mathrm{ng})$ of SB converted

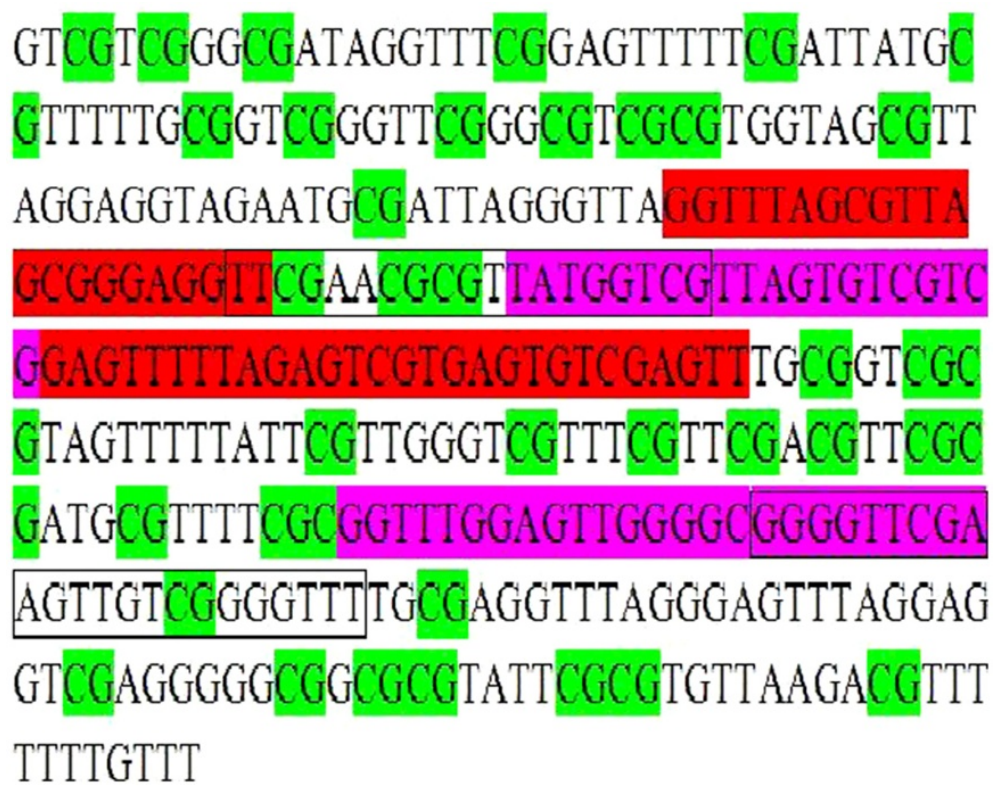

Figure 1 The primers of MS-HRM and MSP assays for CST6 promoter methylation. The MS-HRM primers are shown in red. The outer MSP primers are framed, while the inner ones are shown in purple. The region from -162 up to +188 is depicted (+1 is the Transcriptional start site). Note that this sequence is produced after bisulfite conversion of genomic DNA. All CpGs are considered to be methylated, and therefore are unaffected during the conversion process. 
DNA was added in the PCR reaction mix, which consisted of 1X PCR Buffer (Invitrogen, USA), $0.4 \mathrm{mM}$ for each dNTP (Invitrogen, USA), $0.05 \mathrm{U} / \mu \mathrm{L}$ Platinum ${ }^{\circledR} \mathrm{Taq}$ DNA Polymerase (Invitrogen, USA), $0.25 \mu \mathrm{g} / \mu \mathrm{L}$ BSA (Sigma, Germany), 1X LCGreen Plus Dye (Idaho Technology, USA), $0.25 \mu \mathrm{M}$ primers, and $\mathrm{Mg}^{2+}(2.5 \mathrm{mM})$. $\mathrm{dH}_{2} \mathrm{O}$ was used to supplement up to $10 \mu \mathrm{L}$. The realtime PCR protocol began with one cycle at $95^{\circ} \mathrm{C}$ for 5 min followed by 50 cycles of: $95^{\circ} \mathrm{C}$ for $15 \mathrm{~s}, 60^{\circ} \mathrm{C}$ for $10 \mathrm{~s}$ and $72^{\circ} \mathrm{C}$ for $20 \mathrm{~s}$. Immediately after amplification, a re-annealing cycle consisting of $95^{\circ} \mathrm{C}$ for $1 \mathrm{~min}$ and a rapid cooling to $70^{\circ} \mathrm{C}$ for 1 min was introduced in order to prepare the melting curve acquisition step. Real-time fluorescence acquisition was set at the elongation step $\left(72^{\circ} \mathrm{C}\right)$. Samples whose amplification begun late or the relative fluorescence value on the raw melting-curve plot was low were not further processed. All PCR reactions were performed in triplicate for each sample.

\section{High resolution melting analysis}

All assay optimization studies were performed first in the HR-1 High Resolution Melter (Idaho Technology, USA). For this reason, Real-time PCR was performed in the LightCycler 2.0 instrument using glass capillary tubes that were transferred after PCR to the HR-1 High Resolution Melter. Melting data acquisition began at $69^{\circ} \mathrm{C}$ and ended in $95^{\circ} \mathrm{C}$, using a ramp rate of $0.3^{\circ} \mathrm{C} / \mathrm{s}$. High Resolution Melting Analysis was also performed in the LightCycler ${ }^{\circledR} 480$ instrument (Roche Applied Science, Germany) using 96-well plates (Roche Applied Science, Germany). Data processing included normalization, and resulted on the normalized melting curves and the respective negative derivative of fluorescence over the temperature plots, using the LightCycler $480^{\circledR}$ gene scanning software. The settings for data collection were 50 fluorescence acquisition points per degree centigrade resulting on a ramp rate of $0.01^{\circ} \mathrm{C} / \mathrm{s}$. Comparison of the melting curve or the peaks of an unknown sample with those of the controls gave the semi-quantitative estimation for the methylation level of that sample.

\section{Results}

\section{Assay optimization}

Fully methylated and fully unmethylated DNA, as well as synthetic methylated DNA mixtures were used as controls for the optimization of the assay conditions, and evaluation of the analytical sensitivity and specificity of the MS-HRMA assay.

\section{Annealing temperature}

Three different annealing temperatures were tested $\left(60^{\circ} \mathrm{C}\right.$, $61^{\circ} \mathrm{C}$, and $63^{\circ} \mathrm{C}$ ). The normalized melting curves and the respective derivative plots, as obtained for the synthetic methylated DNA mixtures in all these three temperatures, were readily distinguishable from each other at $60^{\circ} \mathrm{C}$ (Additional file 1 Figure S1).

\section{Analytical sensitivity and specificity}

The developed MS-HRMA assay for CST6 methylation is highly specific for SB treated DNA since under these experimental conditions only SB treated DNA is amplified. When genomic DNA isolated from the A13 cell line that was not SB modified was added, amplification under the same conditions was not observed (Additional file 2: Figure S2). We could readily discriminate between SB treated methylated and SB treated unmethylated controls and no dimers or "non-specific" products were observed. As can be seen in Figure 2A the unmethylated and the fully methylated SB treated DNA controls gave only one peak at their expected Tm values respectively. To evaluate the analytical sensitivity of the assay, dilutions of fully methylated to fully unmethylated DNA $(1 \%-100 \%)$ were assessed. The synthetic mixtures appeared having both peaks as expected. Fluorescence difference plots were generated and the ability to discriminate melting transitions of methylated DNA samples from that of unmethylated DNA samples was assessed. As can be seen in Figure 2B the presence of $1 \%$ of methylated CST6 sequence can be easily verified in the presence of 99\% unmethylated CST6 sequence. When the analysis for the same control samples was repeated three times in three different days, melting curves were highly reproducible (Figure 2A).

Before applying the developed methodology in a highthroughput format, we compared our results for the same control samples using both a 96-well plate format LightCycler 480 (II) instrument (Roche, Germany), and the HR-1 instrument. Melting transitions presented almost identical profiles for both instruments (data not shown).

\section{Pilot testing group}

In the pilot testing group, we analyzed by MS-HRMA for CST6 methylation 10 paired breast cancer and 10 adjacent non-cancerous (histologically normal) tissues, 7 histologically cancer-free specimens obtained from healthy women during reduction mammoplasty, and 9 breast fibroadenomas (included as a separate benign tumor group). The methylation levels ranged from slightly lower than $1 \%$ up to approximately $50 \%$. It is interesting to note that in the 10 paired breast cancer and 10 adjacent non-cancerous (histologically normal) tissues studied, in all cases where the tumor sample was found negative for methylation, the adjacent noncancerous tissue was also negative (Additional file 3: Table S1). In two cases, where the tumor samples were methylated at low percentage the adjacent noncancerous tissue were also negative. Among the 10 


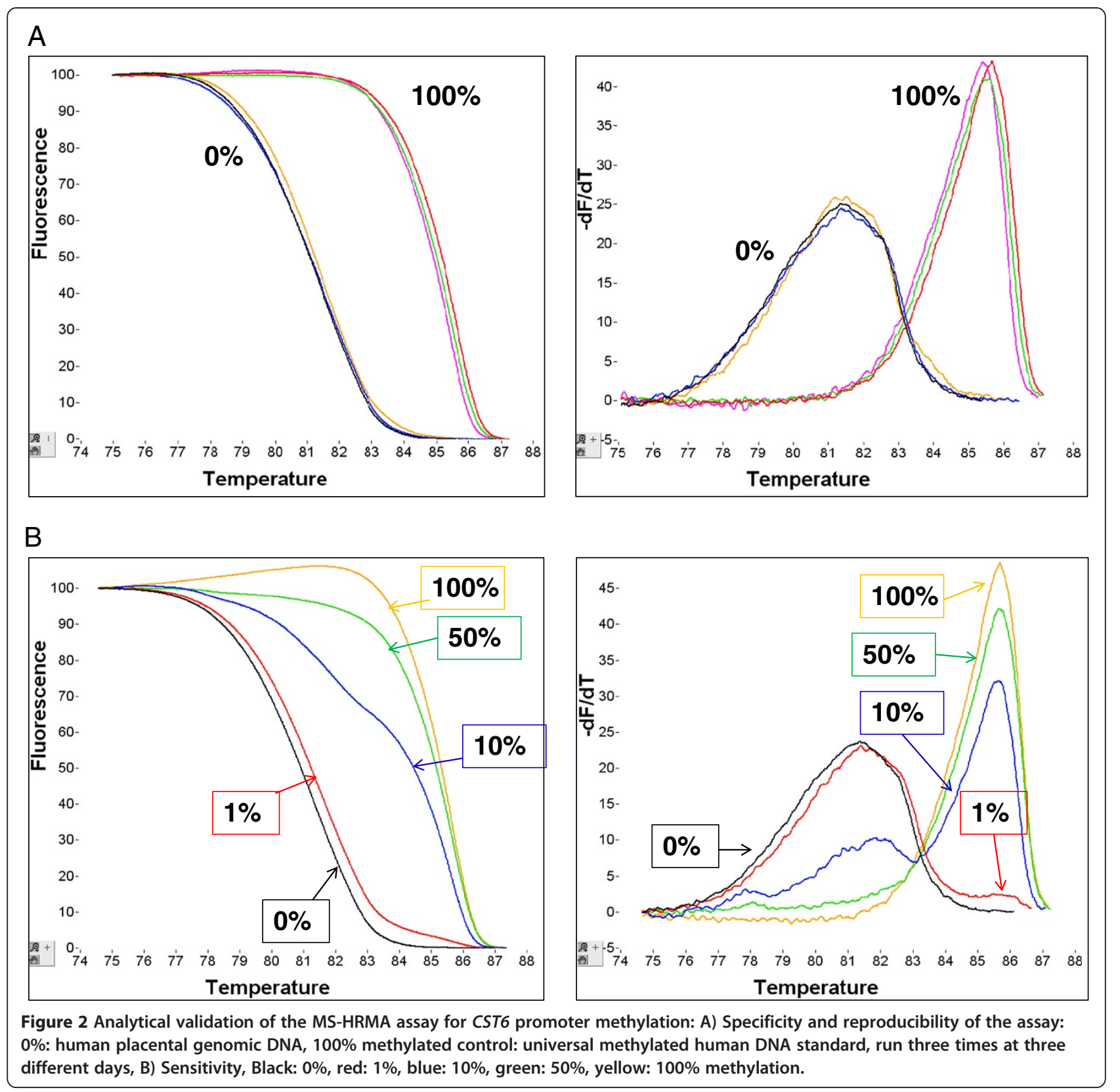

adjacent to tumors non-cancerous (histologically normal) tissues tested only one was found to be methylated. It must be noted that especially in this case, the corresponding tumor sample was heavily methylated (approximately 50\%), and the respective adjacent to the tumor sample showed only $1 \%$ methylation. None of the 7 (0\%) histologically cancer-free specimens from reduction mammoplasty was found to be methylated for CST6 promoter. However, one out of 9 fibroadenomas (11.1\%) showed approximately $10 \%$ methylation for CST6 promoter. Moreover, there was a very good concordance between MS-HRMA and MSP, since in 18/20 (90\%) of these samples MS-HRMA gave the same results as MSP.
There were only 2 samples, where MS-HRMA gave negative results while MSP was positive.

\section{Independent group}

We further applied the developed MS-HRMA assay to evaluate the CST6 methylation status in an independent cohort consisting of 80 FFPE breast carcinomas samples. 39 out of the 80 tumor samples $(48.75 \%)$ were found to be methylated. As can be seen in Figure 3, the melting patterns of the samples when compared to that of the spiked control samples with known percentages of CST6 methylation, always run in parallel, allowed for their classification as non methylated or methylated, while the 


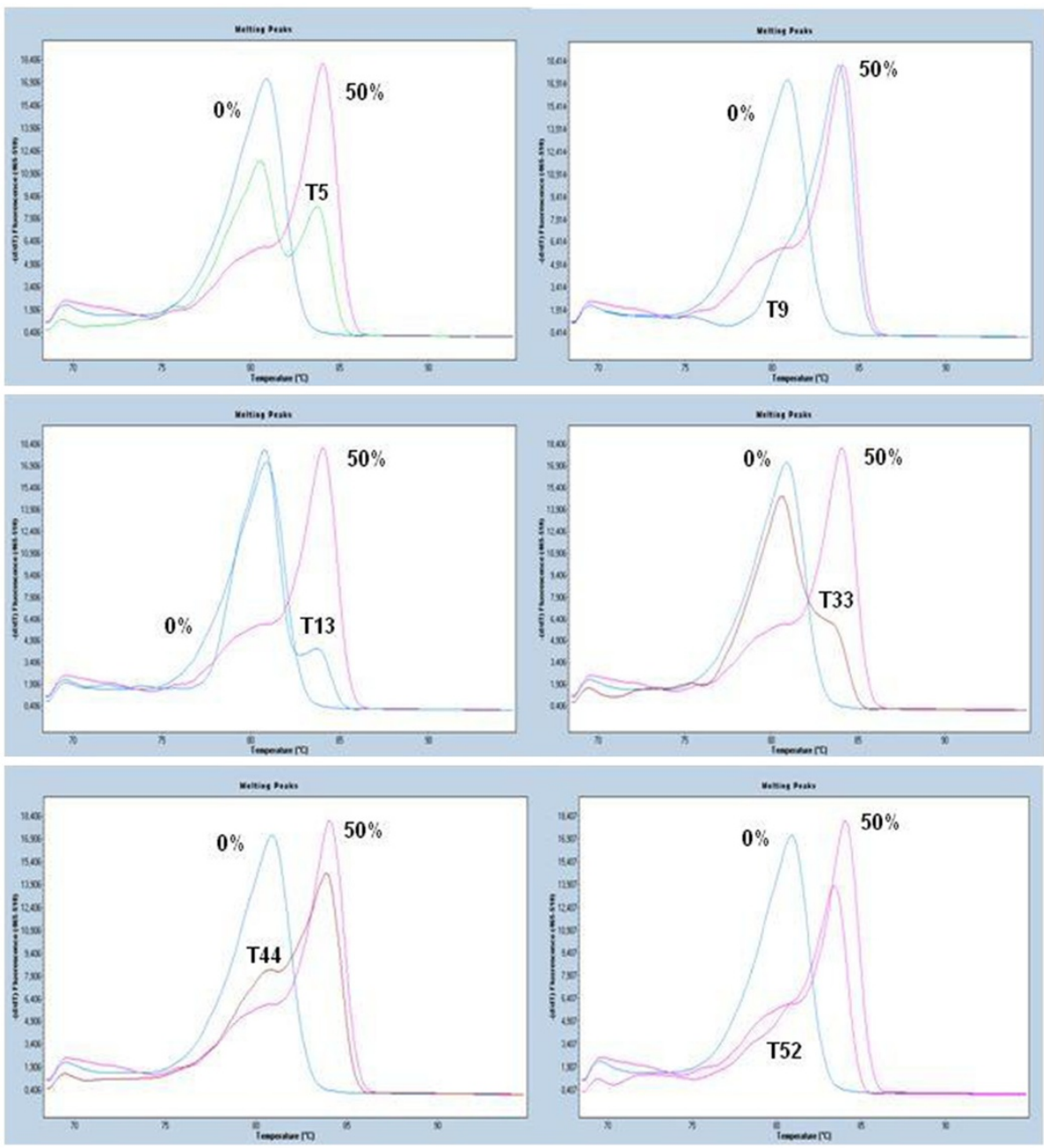

Figure 3 Characteristic first derivative MS-HRMA plots for the semi-quantitative estimation of $\%$ methylation for CST6 promoter methylation by MS-HRMA in six tumor FFPE samples: T5:10\%, T9: 50\%, T13: 1\%, T33: 5\%, T44: 20\% and T52: 20\%. Blue: 0\%, Red: 50\%.

percentage of methylation could also be determined for the latter ones. The clinicopathological characteristics in respect to the methylation status of CST6 of these patients are shown in Table 1. As can be seen in Table 1 there was no correlation between CST6 methylation status and any clinicopathological parameter studied.

Finally, a graph presenting the methylation percentage of each sample across various sample categories, is shown in Figure 4. Mann-Whitney test was performed to evaluate whether a significant difference in methylation levels between those groups exist. As can be seen in this figure, the methylation levels for these 80 tumor FFPE samples were significantly different than those of the 10 non-cancerous adjacent to tumor tissues, and the 7 non-cancerous samples, belonging to healthy persons that underwent mammoplasty surgery, while there was not a significant difference between these samples and the 10 tumors of the independent group as well as with the 9 fibroadenomas tested, since one of them was highly methylated (10\%). Nevertheless, the small number of available fibroadenomas and normal samples do not allow us to have a clear view in respect to those two categories.

\section{Comparison between MS-HRMA assay and MSP}

In the pilot testing group, when all samples were also analyzed by our previously reported MSP assay [26] we found comparable results between the two assays. More specifically, 29 samples were found negative and 5 samples were found positive by both assays, while only 2 samples were positive for MSP and negative for MS-HRMA and no sample was positive by MS-HRMA and negative by MSP. In the independent group, when all these samples were also analyzed by our previously reported MSP assay [26] we also found comparable results between the two assays. More specifically, 21 samples were found negative and 29 samples were found positive by both assays, while 20 samples were positive 
Table 1 Association of CST6 methylation status with clinicopathological features for the independent group $(\mathbf{n}=\mathbf{8 0})$

\begin{tabular}{|c|c|c|c|c|c|}
\hline \multicolumn{2}{|c|}{ Clinicopathological features } & \multicolumn{4}{|c|}{ CST6 methylation } \\
\hline & & $n$ & $+(\%)$ & $-(\%)$ & P value* \\
\hline \multirow[t]{2}{*}{ Age (years) } & $<55$ & 42 & $22(52.4)$ & $20(47.6)$ & 0.495 \\
\hline & $\geq 55$ & 38 & $17(44.7)$ & $21(55.3)$ & \\
\hline \multirow[t]{2}{*}{ Menopausal status } & Pre & 34 & $18(52.9)$ & $16(47.1)$ & 0.519 \\
\hline & Post & 46 & $21(45.7)$ & $25(54.3)$ & \\
\hline \multirow[t]{3}{*}{ Tumor size (cm) } & $0-2.0$ & 23 & $11(47.8)$ & $12(52.2)$ & 0.682 \\
\hline & $2.1-5.0$ & 48 & $22(45.8)$ & $26(54.2)$ & \\
\hline & $>5.0$ & 8 & $5(62.5)$ & $3(37.5)$ & \\
\hline \multirow[t]{3}{*}{ Axillary lymph node } & 0 & 23 & $9(39.1)$ & $14(60.9)$ & 0.366 \\
\hline & $1-3$ & 28 & $14(50.0)$ & $14(50.0)$ & \\
\hline & $\geq 4$ & 27 & $16(59.3)$ & $11(40.7)$ & \\
\hline \multirow[t]{2}{*}{ Tumor grade } & 1 & 22 & $8(36.4)$ & $14(63.6)$ & 0.172 \\
\hline & II, III & 58 & $31(53.4)$ & $27(46.6)$ & \\
\hline \multirow[t]{2}{*}{ Estrogen receptor } & Positive & 49 & $22(44.9)$ & $27(55.1)$ & 0.386 \\
\hline & Negative & 31 & $17(54.8)$ & $14(45.2)$ & \\
\hline \multirow[t]{2}{*}{ Progesterone receptor } & Positive & 26 & $10(38.5)$ & $16(61.5)$ & 0.201 \\
\hline & Negative & 54 & $29(53.7)$ & $25(46.3)$ & \\
\hline
\end{tabular}

*: Chi-square test.

for MSP and negative for MS-HRMA and 10 samples were positive by MS-HRMA and negative by MSP. In total, for $84 / 116(72.4 \%)$ samples the two methods gave comparable results, (Table 2). More specifically, 50 samples were found negative and 34 samples were found positive by both assays, while 22 samples were positive for MSP and negative for MS-HRMA and 10 samples were positive by MS-HRMA and negative by MSP. For comparison of these two methods we used the Mac Nemar test which is a non-parametric method used on nominal data. According to this test the null hypothesis of marginal homogeneity states that the two marginal propabilities for each method are the same. The resulting $\mathrm{P}$ value using a binomial distribution, $(\mathrm{P}=0.050)$ indicated that the two methods are giving comparable results. Moreover, we have evaluated the agreement between these two methods by calculating the kappa index adjusted for a 2-way comparison. This index that has been developed as a measure of agreement that is corrected for chance and according to the Guidelines for Strength of Agreement Indicated with $\mathrm{K}$ Values, the resulting kappa value of 0.4436 is indicative of a moderate agreement between these two methods [35]. Kappa index was calculated according to a program that is available online (http://vassarstats.net/kappa.html) while statistical analysis was performed using the SPSS Windows version 17.0 (SPSS Inc., Chicago, IL).

\section{Discussion}

Cystatin M, originally described as a putative tumor suppressor, whose expression is often diminished or completely lost in metastatic breast cancers [15] has been clearly shown to be epigenetically regulated by strong hypermethylation of the CST6 gene promoter in breast cancer cell lines [23], in breast cancer and metastatic lesions in the lymph nodes [34], in malignant gliomas [36], in cervical [37] and prostate cancer [38]. Because

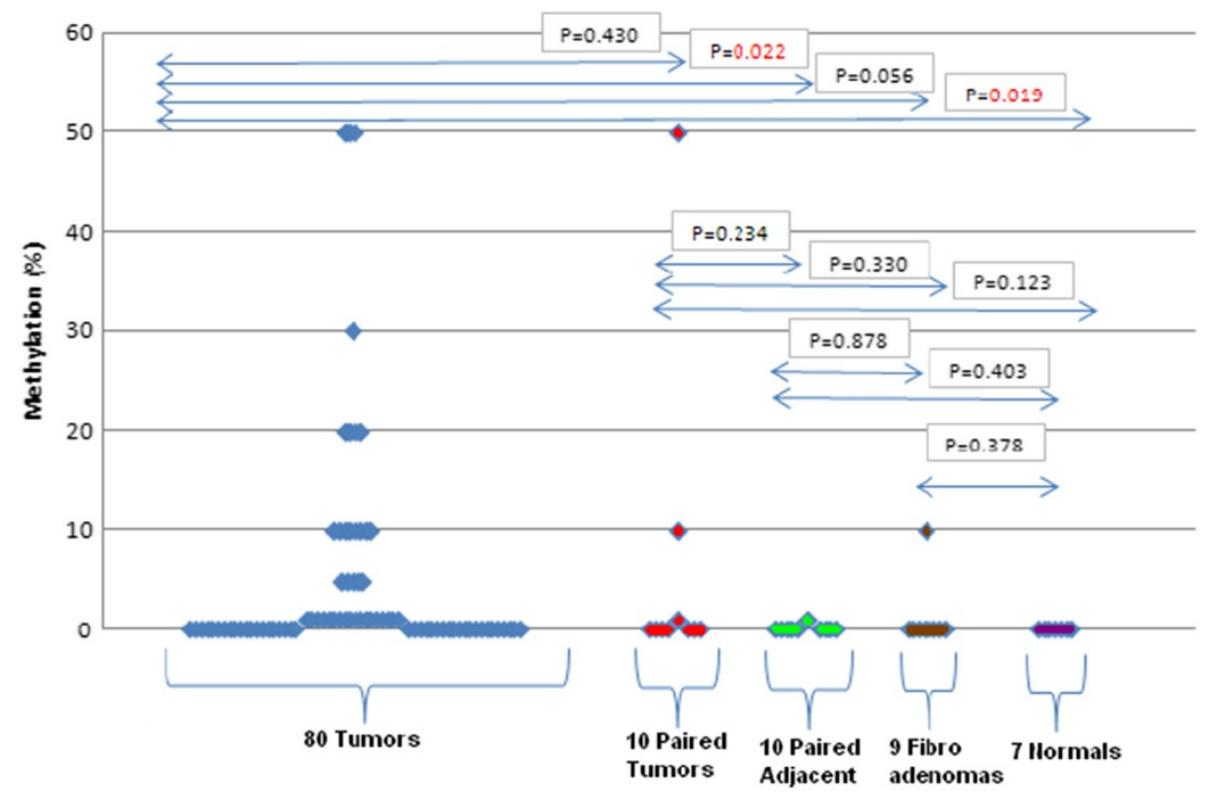

Figure 4 CTS6 methylation levels as estimated by MS-HRMA in the pilot testing group and independent group. $P$ values estimated by the Mann-Whitney test. 


\begin{tabular}{|c|c|c|c|c|}
\hline \multirow[t]{2}{*}{ Method } & & \multicolumn{2}{|l|}{ MSP } & \multirow[t]{2}{*}{ Total } \\
\hline & & pos & neg & \\
\hline \multirow[t]{2}{*}{ MS-HRM } & pos & 34 & 10 & 44 \\
\hline & neg & 22 & 50 & 72 \\
\hline total & & 56 & 60 & 116 \\
\hline \multicolumn{5}{|c|}{ Indices of agreement for MS-HRMA and MSP for CST6 methylation } \\
\hline Agreement index & Type of agreement & Calculated values & Standard error & $\mathrm{Cl}(95 \%)$ \\
\hline$p_{\circ}$ & overall & 0.7241 & & \\
\hline Ppos & positive & 0.6800 & & \\
\hline$p_{\text {neg }}$ & negative & 0.7575 & & \\
\hline$p_{e}$ & chance & 0.5041 & & \\
\hline Kappa index & Chance corrected & 0.4436 & 0.0817 & $0.2834-0.6038$ \\
\hline
\end{tabular}

promoter hypermethylation does not account for the loss of CST6 expression in all tumors alternative modes of CST6 repression are likely, such as histone deacetylation and repressive chromatin structure may be involved [37], since silencing of CST6 has been associated with repressive trimethyl-H3K27 and dimethyl-H3K9 histone marks [39].

Recently, CST6 was also identified among 10 hypermethylated genes that distinguish between cancerous and normal tissues according to the extent of methylation [40]. Moreover, a whole-genome approach using a human gene promoter tiling microarray platform to identify genome-wide and gene-specific epigenetic signatures of breast cancer metastasis to lymph nodes led to functional associations between the methylation status and expression of genes CDH1, CST6, EGFR, SNAI2 and ZEB2 associated with epithelial-mesenchymal transition [41]. In addition, a recent functional epigenetic study of renal cell carcinoma (RCC) cell lines and primary tumors by high-density gene expression microarrays identified CST6 as one of eight genes that showed frequent $(>30 \%)$ tumor-specific promoter region hypermethylation associated with transcriptional silencing (epigenetically inactivated candidate RCC TSGs). According to this study, re-expression of BNC1, CST6, RPRM and SFRP1 suppressed the growth of RCC cell lines [42]. All these recent studies are in support of the importance of CST6 promoter methylation in metastasis.

Our group has shown for the first time the prognostic significance of CST6 promoter methylation in patients with operable breast cancer [26]. According to our findings, the diagnostic sensitivity and specificity of CST6 methylation as a biomarker for prediction of relapses and deaths in operable breast cancer seems to be quite promising [26]. Moreover, we have recently shown that CST6 promoter was methylated in Circulating Tumor Cells (CTC) isolated from peripheral blood of breast cancer patients, in both groups of early disease and verified metastasis [27]. A recent study has also shown that cystatin M loss may be associated with the losses of ER, PR, and HER4 in invasive breast cancer [43].

Based on all these studies, we strongly believe that the reliable and easy detection of CST6 methylation in clinical samples will be of great importance for cancer research. For this reason we decided to develop a closed tube, highly sensitive, cost-effective, rapid and easy-toperform assay for CST6 promoter methylation based on methylation-sensitive high resolution melting analysis (MS-HRMA). Resolution of DNA methylation by melting analysis relies on the fact that the $T \mathrm{~m}$ of a PCR product generated from bisulfite-treated DNA reflects the methylation status of the original DNA template [44]. Because unmethylated cytosines will be converted into uracil during bisulfite treatment and subsequently amplified as thymine, whereas methylcytosines will remain as methylcytosine and be amplified as cytosine, the methylated sequence will have a higher G:C content, and hence a higher $\mathrm{Tm}$, than the corresponding unmethylated sequence. After amplification with primers that will not differentiate between methylated and unmethylated molecules, the melting properties of the PCR products can be examined in the thermal cycler by slowly elevating the temperature under continuous or step-wise fluorescence acquisition. The melting curves or derived melting peaks provide a profile of the methylation status of the entire pool of DNA molecules in the sample [44].

Many reports have already clearly illustrated the great potential of melting analysis for sensitive and highthroughput assessment of DNA methylation in inherited disorders and cancer [6,11-13,30-33,44]. Compared with current gel-based assays MS-HRMA has the important advantage of the closed-tube format, which simplifies the procedure, decreases the risk of PCR contamination, and 
decreases analysis time. In addition, melting analysis resolves heterogeneous methylation, detects methylated and unmethylated alleles in the same reaction, and requires only standard, inexpensive PCR reagents. In addition, the design of individual assays is simple [45-47].

The developed assay is highly specific and sensitive since it can detect the presence of low abundance CST6 methylated DNA sequences (down to 1\%). Moreover to the best of our knowledge, this is the first assay reported so far that provides additionally a semi-quantitative estimation of CST6 promoter methylation. When compared to MSP, the developed MS-HRMA gives comparable but not identical results. The discrepancies between MS-HRMA and MSP can be explained by the different principles on which these methods are based. In MSP we get a positive signal only when the specific CpG island that the primers are designed for is methylated. However it is known that different samples can vary in the methylation sites in specific positions in their CpG islands. In this way if a sample is methylated in positions 3,6 and 7 and the MSP primers are designed to recognize methylation in positions 4,5 and 8 , MSP will give a negative result, while MS-HRMA will give a positive result since it is affected by the presence of any methylated CpG island that is located between the primers. In the opposite way, if the methylation sites that are recognized by the MSP primers are not included in the region amplified by MS-HRMA primers a sample found positive by MSP will be negative by MS-HRMA.

This is the first time that methylation levels for CST6 are reported in clinical samples. Based on our findings, we can definitely say that these levels vary significantly among samples. An interesting finding is that a histologically "non-cancerous" tissue that was adjacent to a highly methylated (50\%) tumor sample was also found to be methylated, at a lower percentage (1\%). CST6 methylation is an early event in breast cancer, since methylation of the CST6 promoter has already been reported in 7 out of 28 corresponding normal tumor-adjacent breast tissues samples [25]. This could possibly indicate that some "normal" cells surrounding the tumor tissue have already a malignant transformation, not detected by conventional immunohistochemistry. In our study we have used whole tissue sections containing more than $80 \%$ of tumour cells. However, we can speculate that the percentage of contaminating normal cells affect the level of methylation seen in our samples. For this reason, we believe that laser capture microdissection could ensure a higher proportion of lesional cells in clinical samples to be studied.

\section{Conclusions}

The developed methylation-sensitive high resolution melting assay (MS-HRMA) for the semi-quantitative determination of CST6 promoter methylation can be a very useful tool to evaluate reliably and semi-quantitatively CST6 methylation in a variety of clinical samples. Moreover it is a closed tube assay, easily applicable in many real time PCR instruments equipped with high resolution melting analysis software, cost-effective, rapid and easy-to-perform. It gives comparable results to MSP in less time, while it offers the advantage of additionally providing an estimation of the level of methylation.

\section{Additional files}

\begin{abstract}
Additional file 1: Figure S1. Optimization of the annealing temperature of the MS-HRMA assay for CST6 promoter methylation. Normalized melting curves and first derivative plots for a) $63^{\circ} \mathrm{C}$ : Black: $0 \%$, red: $1 \%$, blue: $10 \%$, green: $50 \%$, yellow: $100 \%$ methylation b) $61^{\circ} \mathrm{C}$ : Black: $0 \%$, red: $1 \%$, blue: $10 \%$, yellow: $50 \%$, green: $100 \%$ methylation and c) $60^{\circ} \mathrm{C}$ : Black: $0 \%$, red: $1 \%$, blue: $10 \%$, green: $50 \%$, yellow: $100 \%$ methylation.

Additional file 2: Figure S2. Specificity of MS-HRMA assay for CST6 promoter methylation: PCR products of the SB modified positive controls and genomic DNA (unconverted). 1) DNA ladder 2) negative control $\left(\mathrm{H}_{2} \mathrm{O}\right)$, 3) $0 \%$ methylated control 4) $1 \%$ methylated control 5) $10 \%$ methylated control 6) 50\% methylated control 7) 100\% methylated control 8) genomic DNA (unconverted).

Additional file 3: Table S1. CST6 methylation status in 10 paired breast cancer and 10 adjacent non-cancerous tissues as evaluated by both the developed MS-HRMA and MSP [26] assays.
\end{abstract}

\section{Abbreviations}

MSP: Methylation-specific PCR; MS-HRMA: Methylation-sensitive

high-resolution melting analysis; CST6: Cystatin M gene; SB: Sodium bisulfite MIP: methylation independent PCR; HRMA: High-resolution melting analysis; FFPE: Formalin fixed paraffin-embedded; CTC: Circulating tumor cell.

\section{Competing interests}

The authors declare that they have no competing interests.

\section{Authors' contributions}

LD and PV have made substantial contributions to the analysis and acquisition of data, VG has provided the clinical samples and has been involved in drafting the manuscript and EL conceived of the study, and participated in its design and coordination and helped to draft the manuscript and has given the final approval of the version to be published. All authors read and approved the final manuscript.

\section{Acknowledgements}

This work was supported by the Special Account for Research Grants (SARG) of the National and Kapodistrian University of Athens, and the General Secretariat of Research and Technology in Greece.

\section{Author details}

${ }^{1}$ Laboratory of Analytical Chemistry, Department of Chemistry, University of Athens, Athens 15771, Greece. ${ }^{2}$ Department of Medical Oncology, University General Hospital of Heraklion, PO BOX 1352, Crete 71110, Greece. ${ }^{3}$ Present address: Biomolecular Medicine, Department of Surgery and Cancer, Imperial College London, London SW7 2AZ, UK.

Received: 25 April 2012 Accepted: 23 September 2012 Published: 22 October 2012

References

1. Laird PW: The power and promise of DNA methylation markers. Nat Rev Cancer 2003, 3:253-266.

2. Ferguson AT, Evron E, Umbricht CB, Pandita TK, Chan TA, Hermeking H, Marks JR, Lambers AR, Futreal PA, Stampfer MR, Sukumar S: High frequency 
of hypermethylation at the 14.3.3 sigma locus leads to gene silencing in breast cancer. Proc Natl Acad Sci USA 2000, 97:6049-6054.

3. Esteller M: Epigenetics in cancer (Review). N Engl J Med 2008, 358:1148-1159.

4. Jones PA, Baylin SB: The fundamental role of epigenetic events in cancer. Nat Rev Genet 2002, 3:415-428.

5. Esteller M: CpG island hypermethylation and tumor suppressor genes: a booming present, a brighter future. Oncogene 2002, 21:5427-5440.

6. Wojdacz TK, Dobrovic A, Hansen LL: Methylation-sensitive high-resolution melting. Nat Protoc 2008, 3:1903-1908.

7. Wittwer CT, Reed GH, Gundry CN, Vandersteen JG, Pryor RJ: HighResolution Genotyping by Amplicon Melting Analysis Using LCGreen. Clin Chem 2003, 49:853-860.

8. Reed GH, Wittwer CT: Sensitivity and specificity of single-nucleotide polymorphism scanning by high-resolution melting analysis. Clin Chem 2004, 50:1748-1754.

9. Liew M, Pryor R, Palais R, Meadows C, Erali M, Lyon E, Wittwer C: Genotyping of single-nucleotide polymorphisms by high-resolution melting of small amplicons. Clin Chem 2004, 50:1156-1164.

10. Gundry CN, Dobrowolski SF, Martin YR, Robbins TC, Nay LM, Boyd N: Base-pair neutral homozygotes can be discriminated by calibrated high-resolution melting of small amplicons. Nucleic Acids Res 2008, 36:3401-3408

11. Krypuy M, Newnham GM, Thomas DM, Conron M, Dobrovic A: High resolution melting analysis for the rapid and sensitive detection of mutations in clinical samples: KRAS codon 12 and 13 mutations in non-small cell lung cancer. BMC Cancer 2006, 6:295.

12. Zhou L, Wang Y, Wittwer CT: Rare allele enrichment and detection by allele-specific $\mathrm{PCR}$, competitive probe blocking and melting analysis. Biotechniques 2011, 50:311-318.

13. Wojdacz TK, Dobrovic A: Methylation-sensitive high resolution melting (MS-HRM): a new approach for sensitive and high-throughput assessment of methylation. Nucleic Acids Res 2007, 35:e41.

14. Turk $V$, Bode $W$ : The cystatins: protein inhibitors of cystein proteininases. FEBS Lett 1991, 285:213-219.

15. Sotiropoulou G, Anisowicz A, Sager R: Identification, cloning, and characterization of cystatin $M$, a novel cysteine proteinase inhibitor, down-regulated in breast cancer. J Biol Chem 1997 272:903-910.

16. Ni J, Abrahamson M, Zhang M, Fernandez MA, Grubb A, Su J, Yu GL, Li Y, Parmelee D, Xing L, Coleman TA, Gentz S, Thotakura R, Nguyen N, Hesselberg M, Gentz R: Cystatin E is a novel human cysteine proteinase inhibitor with structural resemblance to family 2 cystatins. J Biol Chem 1997, 272:10853-10858.

17. Stenman G, Astrom AK, Roijer E, Sotiropoulou G, Zhang M, Sager R: Assignment of a novel cysteine proteinase inhibitor (CST6) to $11 \mathrm{q} 13$ by fluorescence in situ hybridization. Cytogenet Cell Gent 1997, 76:45-46.

18. Zeeuwen $\mathrm{PL}$, Cheng T, Schalkwijk J: The biology of cystatin M/E and its cognate target proteases. J Invest Dermatol 2009, 129:1327-1338.

19. Cheng T, Hitomi $K$, van Vlijmen-Willems IM, de Jongh GJ, Yamamoto $K$, Nishi K, Watts C, Reinheckel T, Schalkwijk J, Zeeuwen PL: Cystatin M/E is a high affinity inhibitor of cathepsin $V$ and cathepsin $L$ by a reactive site that is distinct from the legumain-binding site. A novel clue for the role of cystatin M/E in epidermal cornification. J Biol Chem 2006, 281:15893-15899.

20. Gawenda J, Traub F, Lück HJ, Kreipe H, von Wasielewski R: Legumain expression as a prognostic factor in breast cancer patients. Breast Cancer Res Treat 2007, 102:1-6.

21. Murthy RV, Arbman G, Gao J, Roodman GD, Sun XF: Legumain expression in relation to clinicopathologic and biological variables in colorectal cancer. Clin Cancer Res 2005, 11:2293-2299.

22. Mohamed MM, Sloane BF: Cysteine cathepsins: multifunctional enzymes in cancer. Nat Rev Cancer 2006, 6:764-775.

23. Ai L, Kim W, Kim TY, Fields CR, Massoll NA, Robertson KD, Brown KD: Epigenetic silencing of the tumor suppressor cystatin $M$ occurs during breast cancer progression. Cancer Res 2006, 66:7899-7909.

24. Rivenbark AG, Jones WD, Coleman WB: DNA methylation-dependent silencing of CST6 in human breast cancer cell lines. Lab Invest 2006, 86:1233-1242
25. Schagdarsurengin U, Pfeifer GP, Dammann R: Frequent epigenetic inactivation of cystatin M in breast carcinoma. Oncogene 2007, 26:3089-3094.

26. Kioulafa M, Balkouranidou I, Sotiropoulou G, Kaklamanis L, Mavroudis D, Georgoulias V, Lianidou ES: Methylation of cystatin M promoter is associated with unfavorable prognosis in operable breast cancer. Int J Cancer 2009, 125:2887-2892.

27. Chimonidou M, Strati A, Tzitzira A, Sotiropoulou G, Malamos N, Georgoulias $V$, Lianidou ES: DNA methylation of tumor suppressor and metastasis suppressor genes in circulating tumor cells. Clin Chem 2011, 57:1169-1177

28. Kroupis C, Christopoulos K, Devetzoglou M, Tsiagas I, Lianidou ES: Asymmetric real-time PCR detection of BRCA1 5382insC mutation by melting curve analysis in the LightCycler. Clin Chim Acta 2008, 390:141-144.

29. Warnecke PM, Stirzaker C, Melki JR, Millar DS, Paul CL, Clark SJ: Detection and measurement of PCR bias in quantitative methylation analysis of bisulphate-treated DNA. Nucleic Acids Res 1997, 25:4422-4426.

30. Wojdacz TK, Borgbo T, Hansen LL: Primer design versus PCR bias in methylation independent PCR amplifications. Epigenetics 2009, 4(4):231-234.

31. Wojdacz TK, Dobrovic A: Melting curve assays for DNA methylation analysis. Methods Mol Biol 2009, 507:229-240.

32. Wojdacz TK, Hansen LL, Dobrovic A: A new approach to primer design for the control of PCR bias in methylation studies. BMC Res Notes. 2008, 1:54.

33. Wojdacz TK, Hansen LL: Reversal of PCR bias for improved sensitivity of the DNA methylation melting curve assay. Biotechniques 2006 41:274, 276-278.

34. Rivenbark AG, Livasy CA, Boyd CE, Keppler D, Coleman WB: Methylationdependent silencing of CST6 in primary human breast tumors and metastatic lesions. Exp Mol Pathol 2007, 83:188-197.

35. Kundel $\mathrm{HL}$, Polansky M: Measurement of observer agreement. Radiology 2003, 228:303-308.

36. Qiu J, Ai L, Ramachandran C, Yao B, Gopalakrishnan S, Fields CR, Delmas AL, Dyer LM, Melnick SJ, Yachnis AT, Schwartz PH, Fine HA, Brown KD, Robertson KD: Invasion suppressor cystatin E/M (CST6): high-level cell type-specific expression in normal brain and epigenetic silencing in gliomas. Lab Invest 2008, 88:910-925.

37. Veena MS, Lee G, Keppler D, Mendonca MS, Redpath JL, Stanbridge EJ, Wilczynski SP, Srivatsan ES: Inactivation of the cystatin E/M tumor suppressor gene in cervical cancer. Genes Chromosomes Cancer 2008, 47:740-754.

38. Pulukuri SM, Gorantla B, Knost JA, Rao JS: Frequent loss of cystatin E/M expression implicated in the progression of prostate cancer. Oncogene 2009, 28:2829-2838.

39. Lin HJ, et al: Breast cancer-associated fibroblasts confer AKT1-mediated epigenetic silencing of Cystatin M in epithelial cells. Cancer Res 2008, 68:10257-10266.

40. Radpour R, Kohler C, Haghighi MM, Fan AX, Holzgreve W, Zhong XY: Methylation profiles of 22 candidate genes in breast cancer using highthroughput MALDI-TOF mass array. Oncogene 2009, 28:2969-2978

41. Rodenhiser DI, Andrews J, Kennette W, Sadikovic B, Mendlowitz A, Tuck AB, Chambers AF: Epigenetic mapping and functional analysis in a breast cancer metastasis model using whole-genome promoter tiling microarrays. Breast Cancer Res 2008, 10:R62.

42. Morris MR, Ricketts C, Gentle D, Abdulrahman M, Clarke N, Brown M, Kishida T, Yao M, Latif F, Maher ER: Identification of candidate tumour suppressor genes frequently methylated in renal cell carcinoma. Oncogene 2010, 29:2104-2117.

43. Ko E, Park SE, Cho EY, Kim Y, Hwang JA, Lee YS, Nam SJ, Bang S, Park J, Kim $\mathrm{DH}$ : Cystatin M loss is associated with the losses of estrogen receptor, progesterone receptor, and HER4 in invasive breast cancer. Breast Cancer Res 2010, 12:R100.

44. Dahl C, Guldberg P: High-Resolution Melting for Accurate Assessment of DNA Methylation. Clin Chem (editorial) 2007, 53:1877-1878.

45. Worm J, Aggerholm A, Guldberg P: In-tube DNA methylation profiling by fluorescence melting curve analysis. Clin Chem 2001, 47:1183-1189. 
46. Guldberg P, Worm J, Grønbæk K: Profiling DNA methylation by melting analysis. Methods 2002, 27:121-127.

47. Dahl C, Grønskov K, Larsen LA, Guldberg P, Brøndum-Nielsen K: A homogeneous assay for analysis of FMR1 promoter methylation in patients with fragile $\mathrm{X}$ syndrome. Clin Chem 2007,

53:790-793.

doi:10.1186/1471-2407-12-486

Cite this article as: Dimitrakopoulos et al: A closed-tube methylationsensitive high resolution melting assay (MS-HRMA) for the

semi-quantitative determination of CST6 promoter methylation in clinical samples. BMC Cancer 2012 12:486.

\section{Submit your next manuscript to BioMed Central and take full advantage of:}

- Convenient online submission

- Thorough peer review

- No space constraints or color figure charges

- Immediate publication on acceptance

- Inclusion in PubMed, CAS, Scopus and Google Scholar

- Research which is freely available for redistribution 